In Crescendo, 2019; 10(1): 137-160

Fecha de recepción: 10 de enero del 2019

Fecha de aceptación: 12 de marzo del 2019

\title{
E-PORTAFOLIO PARA LA GESTIÓN Y LA TOMA DE DECISIONES APLICADO AL EJE DE INVESTIGACIÓN DE LA UNIVERSIDAD TÉCNICA DEL NORTE
}

\author{
E-PORTFOLIO FOR THE MANAGEMENT AND DECISION MAKING \\ APPLIED TO THE RESEARCH AREA OF THE \\ UNIVERSIDAD TÉCNICA DEL NORTE
}

\author{
Marco Carlozama', Lourdes Yépez', \\ Jorge Caraguay ${ }^{1}$
}

\section{RESUMEN}

En las organizaciones la automatización de sus procesos son estrategias básicas para la permanencia dentro de su ámbito de influencia y despliegue de sus productos o servicios. En este contexto la data transaccional del día a día se vuelve un activo importante para las empresas e instituciones, siendo así que, la implementación de sistemas de Business Intelligence (BI) para la toma de decisiones son cada vez más comunes. La presente investigación, pretende adaptar el concepto de E-portafolios como medio integrador de recursos y servicios tecnológicos que ayuden a la gestión y la toma de decisiones del eje de investigación de la Universidad Técnica del Norte (UTN). Para efectos de la investigación se tomó un enfoque cualitativo descriptivo, con revisiones bibliográficas de los principales conceptos del proceso de Data Analisys, en la recolección de información se realizó entrevistas a los stakeholder involucrados con el fin de obtener el modelo de negocio con sus indicadores claves para la gestión y toma de decisiones del eje de Investigación de la UTN. La implementación del E-Portafolio permitió mejorar la gestión del eje de investigación, presentando información alineada a los objetivos estratégicos, además la integración de Business Intelligence permitió transformar los datos existentes en conocimiento orientado al modelo del negocio para la toma de decisiones.

PALABRAS ClAVE: E-Portafolio, Business Intelligence (BI), Almacenes de Datos (DW), Universidad, Indicadores de investigación.

1 Universidad Técnica del Norte. 


\begin{abstract}
In organizations the automation of their processes are basic strategies for permanence within their sphere of influence and deployment of their products or services. In this context, day-to-day transactional data becomes an important asset for companies and institutions, and therefore, the implementation of Business Intelligence (BI) systems for decision making are becoming more common. The present research aims to adapt the concept of E-portfolios as an integrating means of technological resources and services that help the management and decision-making of the research axis of the Universidad Técnica del Norte (UTN). For the purposes of the research, a qualitative descriptive approach was taken, with bibliographic reviews of the main concepts of the Data Analyzes process. In the collection of information, interviews were carried out with the stakeholders involved in order to obtain the business model with its key indicators. For the management and decision making of the research axis of the UTN. The implementation of the EPortfolio allowed to improve the management of the research axis, presenting information aligned to the strategic objectives, as well as the integration of Business Intelligence allowed transforming existing data into knowledge oriented to the business model for decision making.
\end{abstract}

KEY WORDS: E-Portfolio, Business Intelligence (BI), Data Warehouses (DW), University, Research Indicators.

\title{
I. INTRODUCCIÓN
}

El avance de las Tecnologías de Información y Comunicaciones, sin lugar a duda marca el paso a la era del conocimiento y reingeniería de procesos en todos los ámbitos, tanto a nivel de instituciones privadas como públicas.

El Gobierno ecuatoriano por su parte, desde los diferentes organismos de control, fomenta la actualización y mejora de procesos para alcanzar la sociedad del conocimiento, con énfasis en el ámbito educativo a través de las instituciones de Educación Superior, las cuales deben cumplir estándares de calidad y eficiencia tanto en la parte académica como administrativa.

LaUniversidad Técnica del Norte, como ente formador de profesionales de excelencia a nivel regional, mantiene sus procesos académicos y administrativos acorde a los avances tecnológicos, siendo la Dirección de Desarrollo Tecnológico e Informático, antiguamente conocida como Departamento de Informática, quien ejecuta la transformación tecnológica a través del Plan Estratégico Informático futurista, el cual llevará a la institución a consolidar a la universidad como un ejemplo de trabajo en temas informáticos para las demás instituciones educativas del país.

Actualmente, la universidad tiene el $90 \%$ de procesos académicos y administrativos automatizados, por lo que, en la última evaluación institucional, los indicadores de gestión más elevados fueron los relacionados a los temas de automatización y servicios tecnológicos. 
Sin lugar a duda, el tratamiento digital de los procesos ha sido un arduo trabajo tanto para el DDTI como para usuarios finales, quienes manejan día a día cada interfaz de módulo de acuerdo a su cargo o función que desempeña.

El sistema Integrado UTN, es el sistema ERP que ha venido a lograr esa armonía de trabajo, integrando al usuario y operador de cada módulo, los cuales alimentan cada día una gran base de datos con información veraz y confiable. El Centro universitario de investigación ciencia y tecnología es uno de los favorecidos al contar con un módulo específico que automatiza todos los procesos que este genera.

La presente investigación, pretende adaptar el concepto de e-portafolios como medio integrador de recursos, tomando en cuenta que los mismos: "guían la transmisión y proceso de toda la información necesaria para la sincronización de la toma de decisiones" [1] utilizando herramientas de Business Intelligence para elementos claves de negocio del CUICYT, esto presentado mediante una herramienta CRM que permitirá llegar a la satisfacción del usuario final y por ende a la consecución de los objetivos estratégicos trazados para llegar a su visión como Centro Universitario de Investigación, Ciencia y Tecnología de la Universidad Técnica del Norte, todo esto mediante el uso de la base de datos relacional del sistema ERP que la Universidad viene utilizando por más de cuatro años.

El documento se estructura de la siguiente manera: La sección II presenta conceptos básicos utilizados en el trabajo. La sección III presenta una Contextualización de Business Intelligence. En la sección IV se enfoca las soluciones actuales de BI. En la sección V se presenta una revisión de Almacenes de Datos (DW) y la selección de la metodología de desarrollo. En la sección VI se presenta el caso de estudio, Sección VII Análisis de resultados y sección VIII Conclusiones.

\section{CONCEPTOS BÁSICOS}

\section{A. Sistemas de PLANifiCACión DE RECURSOS EMPRESARIALES (ERP).}

Un sistema ERP es una aplicación informática que permite gestionar todos los procesos de negocio de una compañía en forma integrada. Sus siglas provienen del término en inglés ENTERPRISE RESOURCE PLANNING. Este software debe ser parametrizado y adaptado para responder a las necesidades específicas de cada organización. Una vez implementado un ERP permite a los empleados de una empresa administrar los recursos de todas las áreas, simular distintos escenarios y obtener información. La implementación de esta herramienta en 
una empresa o institución conlleva un proceso de transformación y redefinición de sus procesos [2].

\section{B. E-PORTAFOLIO}

El término portafolio deriva de las colecciones de modelos, fotógrafos, arquitectos, diseñadores o artistas, donde se pretende demostrar la calidad o nivel alcanzado en su profesión a través de la integración de una colección de sus trabajos más representativos o sobresalientes. En ese sentido, es difícil ubicar en una fecha, autor o paradigma específico o único, su surgimiento como estrategia para valorar lo que una persona sabe o domina [3]. Sin embargo, Kalz (2005) afirma que el portafolio elaborado por los estudiantes "no es un concepto nuevo", y que ya había sido introducido por el educador francés Celestin Freinet a finales de los años veinte del siglo pasado, aunque también se le identifica con el movimiento de la escuela activa. No obstante, es en años recientes que el portafolio de aprendizaje ha tenido una suerte de renacimiento, que, según este autor, se debe en buena medida al desarrollo y posibilidades que ofrece la tecnología y al interés por el aprendizaje basado en competencias o en la solución de problemas que ocurren en contextos reales. El término portafolio de aprendizaje (de origen francés, portfolio d'apprentissage) también ha sido empleado como sinónimo de dossier o carpeta de aprendizaje, existiendo una serie de controversias respecto a la mejor manera de designarlo, así como en relación con su contenido y estructura" [3].

Si bien es un concepto que se ha venido manejando de una forma más de evaluación y contenedor de evidencias, la investigación presente enmarca otro enfoque, tomando referencia al enunciado de [4] se puede considerar al portafolio como una maleta de viaje donde se la llena con todo lo esencial. Es por esto que el adoptar el término de portafolio para la gestión como un único punto de acceso a la información clave en la administración y toma de decisiones.

\section{CONTEXTUALIZACIÓN DE BUSINESS INTELLIGENCE}

\section{ANTECEDENTES}

La automatización de los procesos de las empresas y organizaciones de las últimas décadas ha ayudado sin lugar a duda a incrementar su eficiencia e innovar sus procesos internos, sus servicios y sus propios productos; siendo tan alto el impacto que ha permitido evolucionar según sus estrategias. El efecto de mantener automatizados tus procesos conlleva a registrar gran cantidad de informa- 
ción, información que conforme pasan los años se ha convertido en activos intangibles más importantes de las empresas, esto según sus modelos de negocios. Siendo así que el término "Inteligencia de Negocios o Business Intelligence" aparece por primera vez por el año de 1958 en un artículo presentado por el investigador de IBM Hans Peter Luhnn titulado "A Business Intelligence System".

En 1988, Giland, adoptado el concepto Business Intelligence (BI) como concepto de actualidad, donde, dio a conocer un modelo sobre funciones de "Inteligencia competitiva en el mundo corporativo" ahí se habló de "conocimiento que podría ser usado por los gestores para adoptar decisiones estratégicas.

Más adelante, Howard Drener (1989), propuso a Business Intelligence como "los conceptos y métodos para mejorar la toma de decisiones empresariales mediante el uso de sistemas basados en hechos de apoyo".

Ya por el año de 1996 el término "Inteligencia de Negocios" se populariza más con un reporte de Gartner Group, donde se establece a BI como una evolución de los sistemas de apoyo a la toma de decisiones que aparecieron en la década de 1960 y desarrollado por los años 80. "Se requiere más que intuición para tomar decisiones correctas basadas en información exacta y actualizada. Las herramientas de reporte, consulta y análisis de datos pueden ayudar a los usuarios de negocios a navegar a través de un mar de información para sintetizar la información valiosa que en él se encuentra - hoy en día esta categoría de herramientas se les llama Inteligencia de Negocios".

Según Gartner (2013), "Business Intelligence suele definirse como la transformación de los datos de la compañía en conocimiento, con el fin de obtener una ventaja competitiva".

\section{DEFINICIÓN}

Una vez investigado muchas definiciones de BI, se concuerda con dos definiciones. La primera está dada por la Corporación Oracle en un artículo del 2009, el articulo enuncia que: "BI, se la define como la combinación de tecnología, herramientas y procesos que me permiten transformar mis datos almacenados en información, esta información en conocimiento y este conocimiento dirigido a un plan o una estrategia comercial. La inteligencia de negocios debe ser parte de la estrategia empresarial, esta le permite optimizar la utilización de recursos, monitorear el cumplimiento de los objetivos de la empresa y la capacidad de tomar buenas decisiones para así obtener mejores resultados." [5]. 
La segunda definición se ha considerado a Gartner (2013), "Business Intelligence suele definirse como la transformación de los datos de la compañía en conocimiento, con el fin de obtener una ventaja competitiva".

Se muestra dos definiciones tomadas desde la perspectiva empresarial y de los analistas de tecnología, en base a esto se define a Business Intelligence (BI) como un conjunto de métodos, técnicas y herramientas que permiten manipular información sensible de las organizaciones, con el fin de transformarla en conocimiento orientado al modelo del negocio que ayude a la toma de decisiones logrando aumentar así su competitividad.

\section{ARQUITECTURA}

Se presenta a continuación un gráfico donde muestra el proceso de análisis y transformación de la Data.

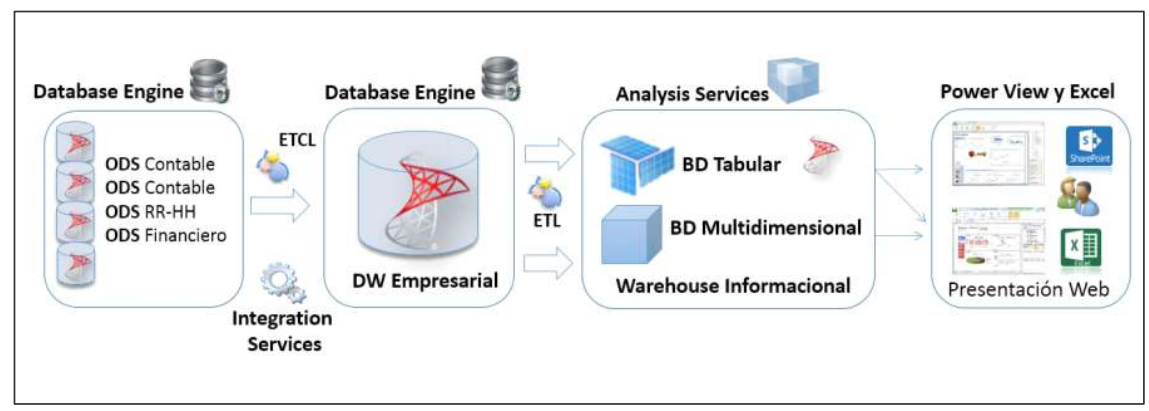

Figura 1. Arquitectura general de la solución BI. [6]

\section{SOLUCIONES ACTUALES DE BI, GARTNER}

El cuadrante mágico de Gartner durante varios años ha provisto de información valiosa para los gerentes de tecnologías a la hora de adquirir o decidirse por alguna u otra herramienta en cuanto a análisis de datos para la toma de decisiones (BI), este se centra en productos que cumplen los criterios de una plataforma moderna de $\mathrm{BI}$ y análisis de datos donde la única recomendación es que las organizaciones deben iniciar sus proyectos de BI utilizando plataformas modernas que logren fomentar la colaboración entre TI y el modelo del negocio a través de un enfoque ágil e interactivo al momento de implementar soluciones tecnológicas. [7]. 
A continuación, se presenta el cuadrante mágico de Gartner el cual aportará para adoptar la herramienta para la solución propuesta.

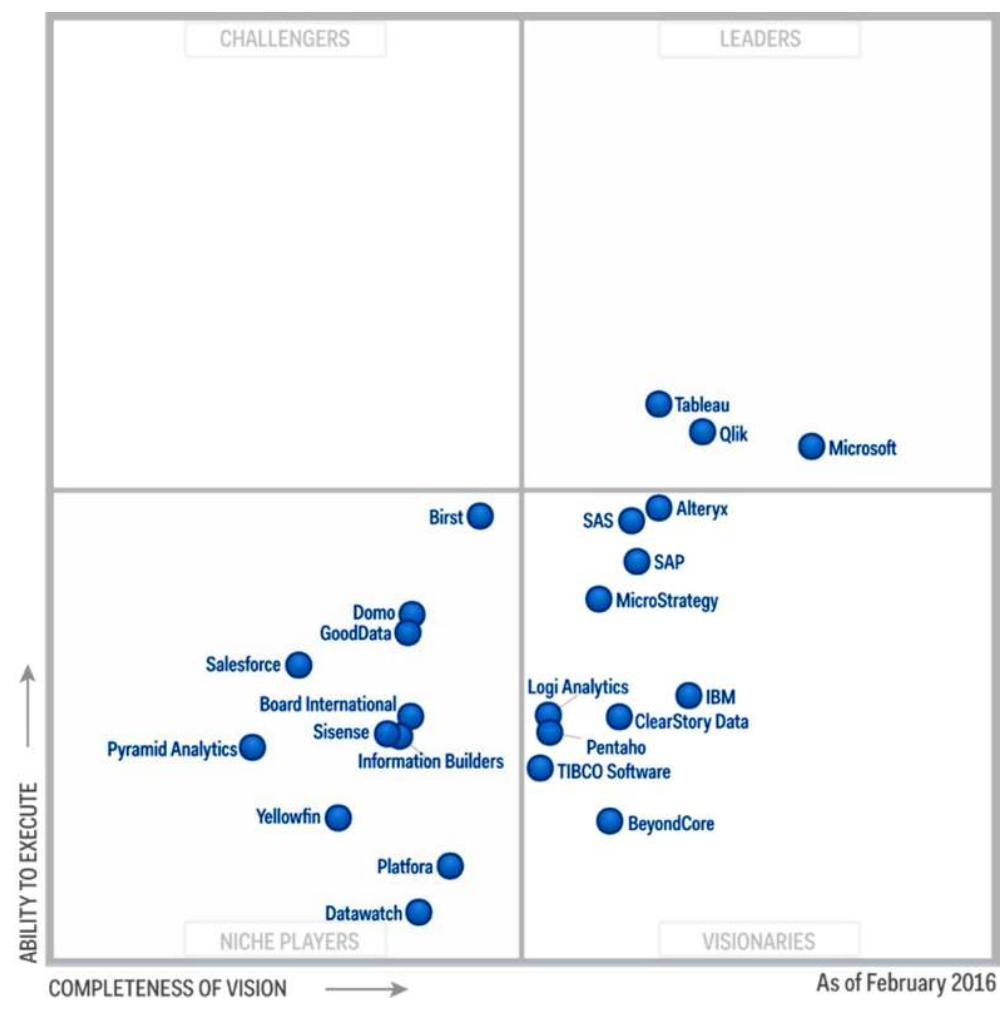

Figura 2. Cuadrante Mágico de Business Intelligence y Analytics Plataformas. Fuente: [8]

A continuación, analizamos la tecnología líder según Gartner en su reporte 2016, Tableu; y la plataforma Oracle Business Intelligence OBI, esta última analizada por ser tecnología ya adquirida por la Institución donde se hará el caso práctico.

\section{TABLEAU}

Tableau se fundamenta o se crea con el propósito de hacer un software de análisis de datos capaz de manejar y presentar información de una manera comprensible para el usuario común. Es un proyecto del Departamento de 
Defensa (DOD) destinado a aumentar la capacidad de las personas para analizar la información. Gracias a la colaboración del Departamento de Ciencias de la Computación de la Universidad de Stanford, el proyecto tomó vuelo con Ph.D Chris Stolte, el mismo que estaba investigando técnicas de visualización para explorar y analizar las bases de datos relacionales y cubos de datos de un DW. [9] Su temprana carrera como programador de bases de datos ayudó a ver los problemas con las herramientas de análisis actuales y sus tendencias.

Tableau es una herramienta de Business Intelligence para el análisis visual de los datos en donde los usuarios pueden crear y distribuir cuadros de mandos interactivos y compartibles que representan las tendencias, variaciones y la densidad de los datos en forma de gráficos y tablas. Tableau puede conectarse a diferentes fuentes de datos como archivos y datos en sistemas relacionales permitiendo hacer una mezcla de ellos y realizar colaboración en tiempo real.

Como una herramienta de visualización de datos Tableau tiene muchas características deseables y únicas, su potente aplicación de descubrimiento y exploración de datos permite contestar preguntas importantes en cuestión de segundos. Puede utilizar la interfaz de arrastrar y soltar de Tableau para visualizar los datos, explorar diferentes puntos de vista, e incluso combinar múltiples bases de datos entre sí con facilidad. No se necesita ninguna secuencia de comandos compleja. Cualquiera que entienda el problema de negocio puede fabricar una visualización de los datos pertinentes [9].

Tableau ofrece soluciones para todo tipo de industrias, departamentos y entornos de datos. Se presenta a continuación las características únicas que le permiten manejar escenarios muy diversos.

Velocidad de Análisis. Tableau no necesita alto nivel de conocimientos de programación, cualquier usuario en su ordenador con acceso a los datos puede comenzar a utilizarlo para obtener valor de los datos.

Autosuficiente. Tableau no necesita una configuración de software complejo. La versión de escritorio que es utilizado por la mayoría de los usuarios se instala fácilmente y contiene todas las características necesarias para iniciar el análisis de datos.

Descubrimiento Visual. El usuario explora y analiza los datos mediante el uso de herramientas visuales como los colores, líneas de tendencia, tablas y gráficos, casi todo lo que se hace es mediante arrastrar y soltar. 
Mezclar diversos conjuntos de datos. Tableau le permite relacionar diferentes fuentes de datos semi-estructurados y sin procesar en tiempo real.

Arquitectura Agnóstico. Tableau funciona en todo tipo de dispositivos. Por lo que el usuario no necesita preocuparse de requisitos de hardware o de software.

Colaboración en tiempo real. En Tableau se puede filtrar, clasificar y analizar los datos sobre la marcha, e incrustar un panel en portales como sitio de SharePoint o Salesforce.

Los datos centralizada. El servidor proporciona una ubicación centralizada para gestionar todas las fuentes de datos publicadas de la organización. Puede eliminar, cambiar permisos, añadir etiquetas y gestionar los horarios en una ubicación conveniente [9].

\section{ORACLE BUSINESS INTELLIGENCE FOUNDATION SUITE}

Se considera como alternativa con alto porcentaje a ser tomada puesto que la Institución donde se aplica la investigación ya cuenta con el licenciamiento de esta herramienta.

Oracle Business Intelligence Foundation Suite ofrece potentes capacidades que dan un valor significativo para las aplicaciones de BI. La suite Oracle Business Intelligence Foundation está compuesta por: Oracle BI Business Intelligence Enterprise Edition (OBIEE), Oracle BI Publisher, Oracle Essbase, Oracle Scorecard y Strategy Management y Oracle Essbase Analytics Link (EAL).

De estas Oracle BI Server y Oracle Essbase son los dos componentes principales del servidor siendo los responsables de la recuperación y federación de datos. Incluye una capa de presentación que ayuda a los usuarios finales.

El servidor de Oracle BI Publisher proporciona la tecnología que ofrece capacidades de generación de informes de gran volumen. Oracle BI Publisher puede acceder a datos de una amplia gama de orígenes de datos a través del modelo de información empresarial común o acceso directo a las tablas físicas y esquemas de los almacenes de datos subyacentes [10].

En el siguiente apartado se describe el proceso de Data Analysis que va desde describir las fuentes de datos a ser transformadas hasta la presentación de información precisa alineada a los objetivos estratégicos y sus necesidades de tal forma que permita tomar decisiones acertadas y permita mejorar la gestión en el caso de estudio propuesto. 


\section{ALMACENES DE DATOS (DW) Y LA SELECCIÓN DE LA METODOLOGÍA DE DESARROLLO}

\section{ALMACÉN DE DATOS (DW)}

Los almacenes de datos (AD), corresponden a la mejora tecnológica en almacenamiento de volúmenes de datos, siendo estos más inteligentes, con más características y funcionalidades. El concepto fundamental de un $\mathrm{AD}$ según W. $\mathrm{H}$. Inmon, considerado el padre de esta disciplina, considera que son sistemas basados en colección de datos orientados a materias, integrado, no volátil, que varía en el tiempo y que están diseñados para procesos de apoyo en la toma de decisiones. [11]

Un almacén de datos es la implementación de una bodega de datos con alcances limitados, a un área específica o bien a la solución de un problema. Esta bodega de datos puede integrarse de dos formas dependiendo de la orientación de la arquitectura que se resuelva implementar.

\section{ENFOQUE TOP-DOWN, LNMON}

En este enfoque se define previamente el AD para luego delimitar en los modelos multidimensionales (MD), el modelo plantea crear el AD que soporte a toda la organización para luego centrarse en cada unidad o departamento que conforma la organización. Es por esto que su principal inconveniente es que es muy costoso en tiempo y recursos por lo que su enfoque no resulta muy adecuado a la hora de diseñar un $\mathrm{AD}$, esto hablando de una forma general, el equipo de desarrollo tomará la mejor orientación según sus necesidades.

\section{ENFOQUE BOTTOM-UP, KIMBALL}

Por el contrario, este enfoque propone crear previamente los MD de cada uno de los departamentos o unidades funcionales de la organización, al no comenzar con una toda esta orientación permite entregar productos en menor tiempo y con menos recursos. En este enfoque el principal problema que se presenta es a la hora de sincronizar las tablas de hechos al momento de hacer la sincronización de los demás MD en un solo AD.

Sin embargo, este enfoque es el más utilizado a la hora de diseñar los AD. 


\section{ANÁLISIS DE METODOLOGÍAS PARA DESARROLLAR ALMACÉN DE DATOS}

Los principales enfoques del diseño del almacén de datos son basados en los mismos datos y en las necesidades. Presentando cada uno de ellos ventajas y debilidades [13]. El enfoque basado en datos analiza la fuente de datos y la remodela para obtener un esquema unidimensional, garantizando la viabilidad del almacén de datos, pero su debilidad es que las necesidades del usuario no se tienen en cuenta. Por otro lado, el enfoque impulsado por los requerimientos considera las metas de negocio para alinear el modelo a la estrategia del negocio para luego producir un esquema multidimensional. Por lo tanto, ese esquema es adherente a las necesidades del usuario, pero puede no ser compatible con la presencia efectiva de datos en la fuente. [14].

El siguiente análisis de metodologías para el diseño de AD pretende identificar cual sería el mejor modelo o bien identificar las mejores orientaciones para fusionar dos o más de ellos. Dedicado a definir una metodología de diseño que integre las ventajas de ambos enfoques.

\section{EL CICLO DE VIDA KIMBALL}

La metodología de desarrollo de AD más utilizada, este propone un marco de trabajo como en la Figura 3, la cual detalla todas las etapas y componentes en todo el proceso de creación del AD.

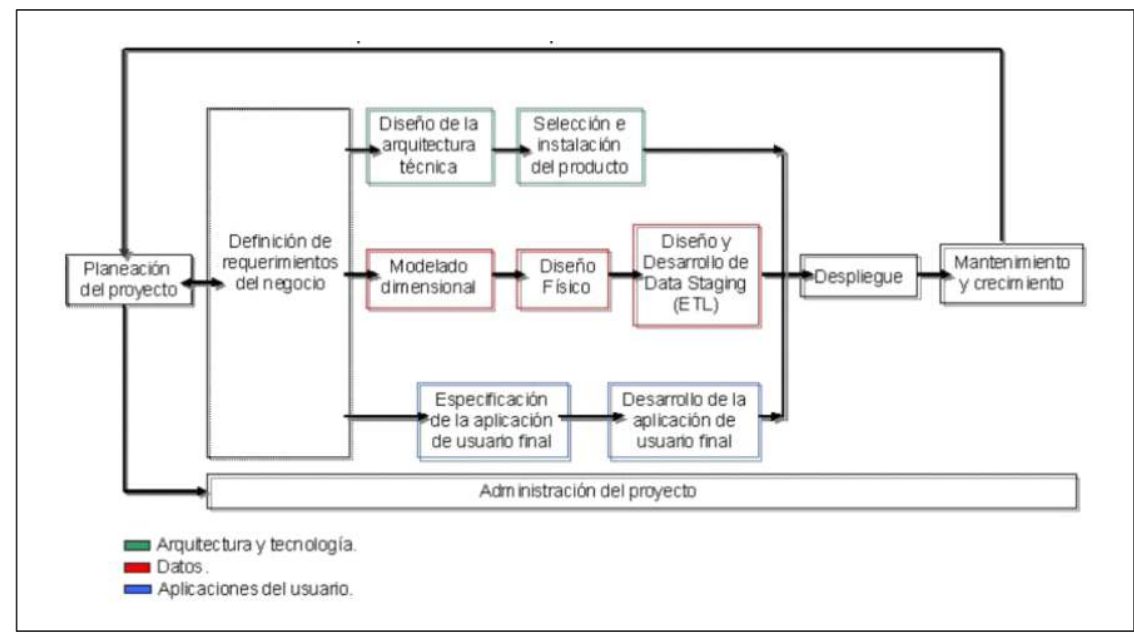

Figura 3. Ciclo de vida para la construcción de un AD según Ralph Kimball. 
La fase de planeación del proyecto, establecer la definición y el alcance del proyecto del $\mathrm{AD}$, incluyendo la valoración y justificación del negocio.

La fase de definición del proyecto es donde se constituye la base relacionada con los datos, la tecnología, y las aplicaciones del usuario.

La ruta roja es la de mayor importancia ya que se asocia con los datos, es donde se realiza el modelado dimensional, en base a los requerimientos levantados de los usuarios.

El diseño físico, es que da las estructuras básicas para soportar el modelado dimensional.

Los ETL en la cual se diseña y desarrollan procesos para extraer, transformar y cargar datos.

Kimball, propone que durante todo el ciclo de vida se debe seguir una administración general del proyecto.

\section{DWEP (DATA WAREHOUSE ENGINEERING PROCESS)}

Un proceso de desarrollo de data warehouse (DW) basado en el Unified Modeling Languagen (UML) y el Proceso Unificado (UP). UP es un proceso genérico y estable en la ingeniería de software. Sus características es que es interactivo e incremental en el proceso de desarrollo de software, se basa principalmente en 4 fases de desarrollo y siete flujos de trabajo. La figura 3 representa claramente la relación entre los flujos de trabajo en los diferentes ámbitos.

DWEP, está basado en componentes, utiliza el UML (Unified Modeling Language - Lenguaje Unificado de Modelado) como lenguaje para modelado lo que significa que es un modelo orientado a objetos por lo que lo hace independiente de cualquier implementación específica ya sea relacional o multidimensional [15].

Para el PU y el DWEP un flujo de trabajo es un conjunto de actividades realizadas en un área determinada cuyo resultado es la construcción de artefactos.

Requerimientos. Los usuarios especifican las medidas y agregaciones más interesantes, el análisis dimensional, el sistema permite obtener los requisitos y funciones para la solución. Además, establece como deben ser las interacciones del sistema.

Análisis. Su objetivo es mejorar la estructura y los requisitos obtenidos en la etapa de requerimientos. 


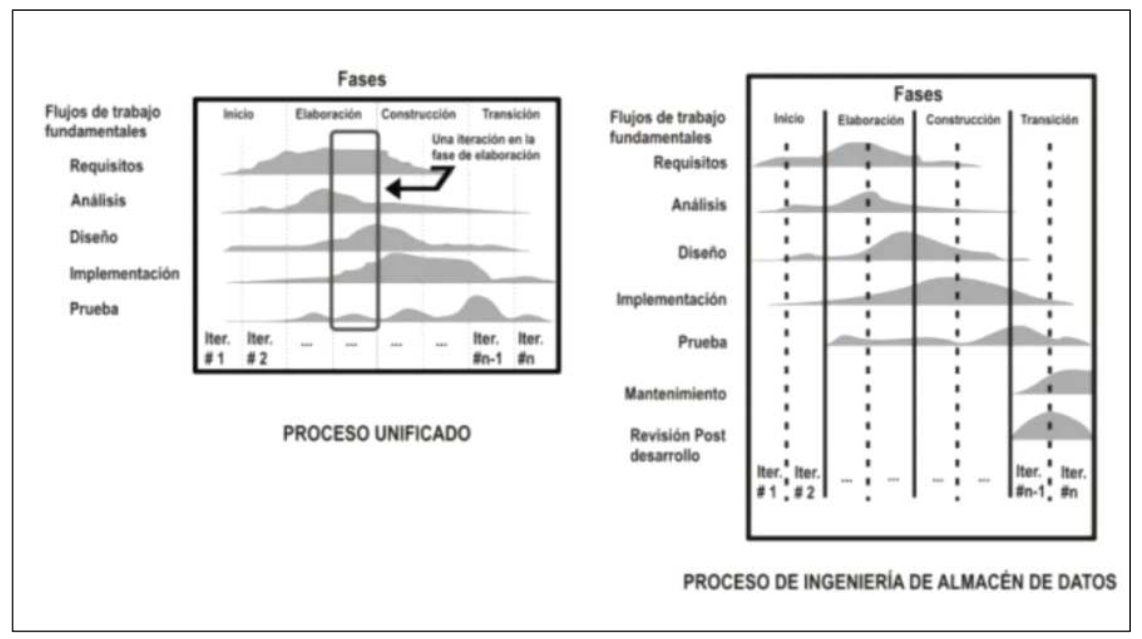

Figura 4. (a) Proceso Unificado y (b) DWEP [15]

Diseño. Como efecto de esta fase se entrega definida la estructura del $\mathrm{AD}$, pero el principal resultado de este flujo de trabajo es el modelo conceptual del AD.

Implementación, durante este flujo de trabajo, el $\mathrm{AD}$ es construido y se empiezan a recibir datos de los sistemas operaciones, se afina para un funcionamiento optimizado, entre otras tareas.

Pruebas, el fin de este flujo de trabajo es verificar que la aplicación funcione correctamente.

Mantenimiento, el objetivo de este flujo de trabajo es definir la actualización y carga de los procesos necesarios para mantener el AD.

Revisiones post desarrollo, no se le considera un flujo de trabajo de las actividades de desarrollo, sino un proceso de revisión para la mejora continua. Se hace seguimiento de las fases en tiempo y esfuerzo con el fin de mejorar el proceso para trabajos futuros. [16].

\section{Enfoque orientado al negocio para el desarrollo del almacén de datos}

Desde una perspectiva empresarial, propone el análisis de la estrategia de negocio, la alineación entre los objetivos del almacén de datos y la estrategia de la organización, realiza el modelado de los requisitos de información orientados a objetivos y obtiene un modelo de almacén de datos multidimensional. Se propor- 
ciona un conjunto de directrices que permiten a los desarrolladores diseñar un almacén de datos alineado con una estrategia empresarial [17].

Se presenta la Figura 5, que describe el proceso de alinear los requisitos al modelo del negocio en el diseño del AD.

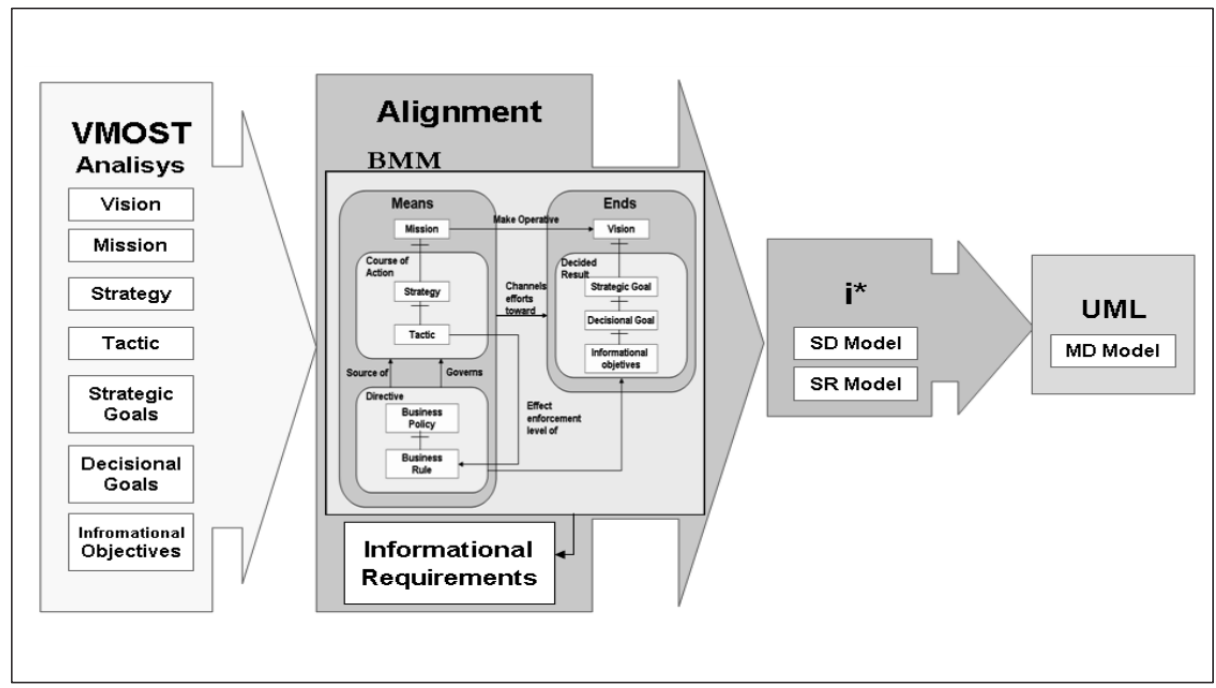

Figura 5. Enfoque al desarrollo de DW orientado a la organización. [17].

Se detalla las fases correspondientes en el modelo propuesto.

\section{ANÁLISIS DE REQUISITOS PARA DW}

La fase de análisis de requerimientos tratar como analizar, comprender y modelar el contexto de negocio, tiene como objetivo representar a los usuarios de DW, los objetivos de negocio de la organización en la que se integra un DW y las relaciones entre el DW y sus usuarios para alcanzar los objetivos de negocio. Esta fase es crucial para el desarrollo de DW, ya que las partes interesadas generalmente no saben cómo describir los requisitos de información y el DW puede no reflejar las necesidades del negocio [17]. Esta fase permite a los desarrolladores situar el DW dentro de su contexto de negocio y relacionarlo con los objetivos empresariales. Los diseñadores pueden entonces obtener más fácilmente lo que los usuarios de DW necesitan hacer con el sistema DW para alcanzar los objetivos de negocio [17]. 


\section{ANÁLISIS DE ESTRATEGIA EMPRESARIAL}

El primer paso en la alineación estratégica de una empresa es establecer su estrategia comercial; El objetivo de una estrategia es definir las metas y objetivos del negocio.

El análisis de VMOST proporciona un medio de reconstruir la estrategia empresarial en los componentes básicos de visión, misión, metas, estrategias, objetivos y tácticas. Ayuda a entender cómo se relacionan estos componentes con la respuesta de un analista a una serie de preguntas clave presentadas [17].

\section{ALINEACIÓN USANDO BMM}

El BMM es la especificación de OMG para modelar los elementos del plan de negocios. Estos motivan y proveen gobierno y orientación empresarial. El modelo BMM ayuda a modelar los sistemas de organización para que se alineen con el negocio y proporcionen apoyo a la motivación empresarial de una organización.

BMM proporciona un vocabulario de negocios que puede ser entendido por las partes interesadas del negocio para desarrollar, comunicar y administrar planes de negocio de una manera organizada [18].

\section{MODELO DE OBJETIVOS PARA DW}

El lenguaje de modelado i * representa los actores, las dependencias y las relaciones con los objetivos a alcanzar de acuerdo con una estrategia empresarial.

Esta técnica consiste en dos modelos: la dependencia estratégica (SD) para describir las relaciones de dependencia entre los diferentes actores dentro de su contexto organizacional y el modelo de racionalidad estratégica (SR) que se utiliza para describir los intereses y preocupaciones de cada actor. Los conceptos centrales en los modelos $\mathrm{i}$ * son las intenciones de los actores y sus relaciones de dependencia para lograr sus objetivos. [18]

\section{CASO DE ESTUDIO: E-PORTAFOLIO PARA LA GESTIÓN Y LA TOMA DE DECISIONES APLICADO AL EJE DE INVESTIGACIÓN DE LA UNIVERSIDAD TÉCNICA DEL NORTE}

Para el desarrollo de la herramienta BI, como propuesta a la solución del problema planteado, se adoptará los paquetes o software de desarrollo e implementación de Data Werehouse y Business Intelligence de la casa del fabricante 
Oracle. Se adopta estas herramientas por su efectividad y confianza que brinda la marca a la hora de desarrollar una aplicación de BI, se considera que la confiabilidad y seguridad de los datos tratados, el respaldo de la marca con su acompañamiento y colaboración en temas puntuales de las herramientas, todos estos elementos son considerados como factores claves para el éxito del proyecto de BI.

Otros factores importantes que ayudaron a decidir con qué herramientas se trabajaría, es el hecho de que la institución tiene licenciado la suite ORACLE y el personal de la Dirección de Desarrollo Tecnológico e Informático (DDTI) viene ya trabajando por alrededor de 10 años con estas herramientas por lo que es fundamental que se siga la línea trazada por el DDTI con su administración actual.

Para cubrir todo el ciclo de vida del aplicativo propuesto, se determinará como guía metodológica a la propuesta por Ralph Kimball, esta metodología es a menudo conocida como modelado Dimensional, es un proceso que asegura una exitosa creación de una fuente de datos, integrada, no volátil y variable en el tiempo que ayudará con la toma de decisiones. El enfoque que tiene la metodología en la construcción del Data Marts es Botton-Up, la cual básicamente lleva cada proceso relevante del negocio de manera incremental.

La arquitectura propuesta hace más énfasis al proceso de Data Analisys, entendiéndose a este concepto como un proceso de tratamiento de los datos de tal forma que permita descubrir información útil que pueda ser utilizada para la toma de decisiones.

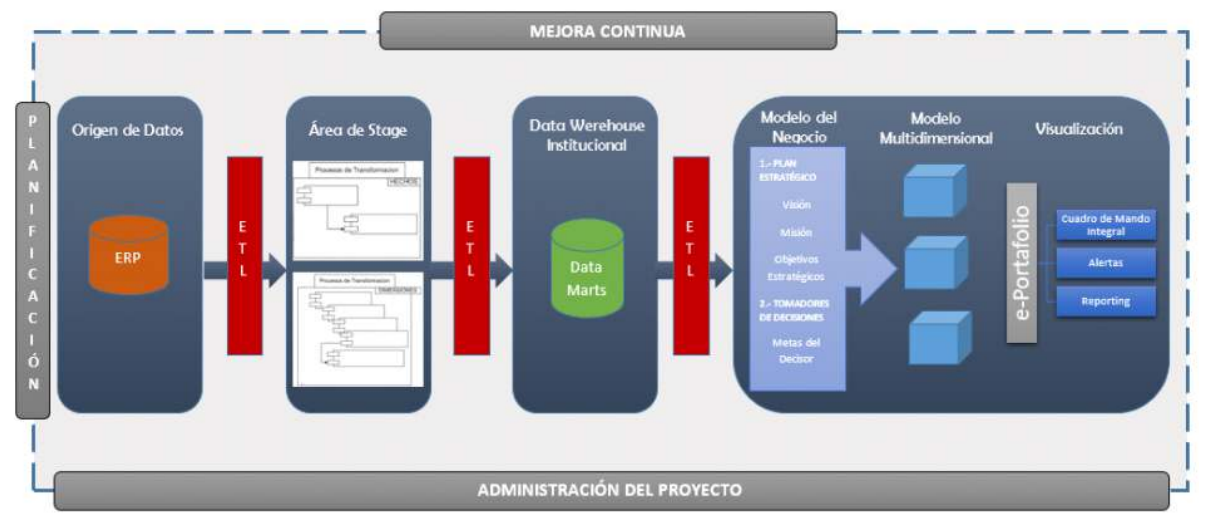

Figura 6. Arquitectura propuesta. 
En el levantamiento de requerimientos se toma dos enfoques que son:

- Plan Estratégico, modelado de estrategia departamental (CUICYT).

- Tomadores de decisiones, modelado las metas del decisor y requerimientos funcionales del sistema.

\section{PORTAFOLIO DE LA DIRECCIÓN DEL CUICYT}

Tabla 1

REQUERIMIENTO FUNCIONAL: AUTENTIFICACIÓN

DE USUARIO

\begin{tabular}{|l|l|}
\hline $\begin{array}{l}\text { Identificación del } \\
\text { requerimiento: }\end{array}$ & RF03 \\
\hline $\begin{array}{l}\text { Nombre del } \\
\text { Requerimiento: }\end{array}$ & Autentificación de Usuario. \\
\hline Características: & Los usuarios deberán identificarse para acceder al sistema. \\
\hline $\begin{array}{l}\text { Descripción del } \\
\text { requerimiento: }\end{array}$ & $\begin{array}{l}\text { El sistema permitirá el acceso de la Directora del CUICYT y } \\
\text { Coordinadores de Investigación de cada Facultad }\end{array}$ \\
\hline $\begin{array}{l}\text { Prioridad del requerimiento: } \\
\text { Alta }\end{array}$ \\
\hline
\end{tabular}

Tabla 2

REQUERIMIENTO FUNCIONAL: HERRAMIENTA BI CON LOS INDICADORES CLAVES DE GESTIÓN DEL CUICYT

\begin{tabular}{|l|l|}
\hline $\begin{array}{l}\text { Identificación del } \\
\text { requerimiento: }\end{array}$ & RF04 \\
\hline $\begin{array}{l}\text { Nombre del } \\
\text { Requerimiento: }\end{array}$ & $\begin{array}{l}\text { Herramienta BI con los indicadores claves de gestión } \\
\text { del CUICYT }\end{array}$ \\
\hline Características: & $\begin{array}{l}\text { El sistema presentará un Dashboard con la información de } \\
\text { los indicadores claves de Investigación. }\end{array}$ \\
\hline $\begin{array}{l}\text { Descripción del } \\
\text { requerimiento: }\end{array}$ & $\begin{array}{l}\text { Como primera pantalla el sistema presentará un Dashboard } \\
\text { con la información de los indicadores claves de Investigación } \\
\text { los mismos que permitirán hacer drill-down de la información } \\
\text { permitiendo llegar a niveles de detalle según permita el } \\
\text { modelo de BI. }\end{array}$ \\
\hline $\begin{array}{l}\text { Prioridad del requerimiento: } \\
\text { Alta }\end{array}$
\end{tabular}


Tabla 3

REQUERIMIENTO FUNCIONAL: INFORMES DINÁMICOS DE PROYECTOS DE INVESTIGACIÓN

\begin{tabular}{|l|l|}
\hline $\begin{array}{l}\text { Identificación del } \\
\text { requerimiento: }\end{array}$ & RF05 \\
\hline $\begin{array}{l}\text { Nombre del } \\
\text { Requerimiento: }\end{array}$ & Informes Dinámicos de Proyectos de Investigación. \\
\hline Características: & $\begin{array}{l}\text { El sistema presentará en el menú de "Investigación" un } \\
\text { submenú "Proyectos de Investigación", donde se presentará } \\
\text { un buscador con varias opciones de búsqueda, el cual } \\
\text { desplegará los campos solicitados por la Dirección del } \\
\text { CUICYT. }\end{array}$ \\
\hline $\begin{array}{l}\text { Descripción del } \\
\text { requerimiento: }\end{array}$ & $\begin{array}{l}\text { El sistema permitirá la búsqueda de proyectos de investiga- } \\
\text { ción de acuerdo a uno o varios parámetros como: convocato- } \\
\text { ria, año, facultad, carrera, área de investigación, línea de } \\
\text { investigación o por Docente. }\end{array}$ \\
\hline $\begin{array}{l}\text { Prioridad del requerimiento: } \\
\text { Alta }\end{array}$
\end{tabular}

Tabla 4

REQUERIMIENTO FUNCIONAL: INFORMES DINÁMICOS DE PRODUCCIÓN CIENTÍFICA

\begin{tabular}{|l|l|}
\hline $\begin{array}{l}\text { Identificación del } \\
\text { requerimiento: }\end{array}$ & RF06 \\
\hline $\begin{array}{l}\text { Nombre del } \\
\text { Requerimiento: }\end{array}$ & Informes Dinámicos de Producción Científica. \\
\hline Características: & $\begin{array}{l}\text { El sistema presentará en el menú de "Investigación" un } \\
\text { submenú "Obras publicadas ", donde se presentará un } \\
\text { buscador con varias opciones de búsqueda, el cual } \\
\text { desplegará los campos solicitados por la Dirección del } \\
\text { CUICYT. }\end{array}$ \\
\hline $\begin{array}{l}\text { Descripción del } \\
\text { requerimiento: }\end{array}$ & $\begin{array}{l}\text { El sistema permitirá la búsqueda de obras publicadas de } \\
\text { acuerdo a uno o varios parámetros como: año, filiación UTN, } \\
\text { facultad, carrera, área de investigación, línea de investigación, } \\
\text { Base de Datos Indexada oporDocente. }\end{array}$ \\
\hline $\begin{array}{l}\text { Prioridad del requerimiento: } \\
\text { Alta }\end{array}$
\end{tabular}


Tabla 5

REQUERIMIENTO FUNCIONAL: MÓDULO DE EVALUACIÓN

DE OBRAS REGISTRADAS

\begin{tabular}{|l|l|}
\hline $\begin{array}{l}\text { Identificación del } \\
\text { requerimiento: }\end{array}$ & RF07 \\
\hline $\begin{array}{l}\text { Nombre del } \\
\text { Requerimiento: }\end{array}$ & Módulo de Evaluación de Obras Registradas. \\
\hline Características: & $\begin{array}{l}\text { El sistema en el Menú "Gestión" se añade el submenú } \\
\text { "Evaluación de Obras registradas", cuya función principal es } \\
\text { facilitar la evaluación de cada obra científica. El coordinador } \\
\text { aceptará o enviará a actualizar el registro de la obra que tenga } \\
\text { novedades. }\end{array}$ \\
\hline $\begin{array}{l}\text { Descripción del } \\
\text { requerimiento: }\end{array}$ & $\begin{array}{l}\text { El sistema permitirá a cada coordinador de investigación } \\
\text { evaluar las obras registradas de su Facultad. Se presentará un } \\
\text { listado de las obras y sus participantes donde el evaluador } \\
\text { podrá verificar cada item subido de la obra. El evaluador } \\
\text { según las novedades encontradas, el sistema le permitirá } \\
\text { enviar un correo electrónico al o los responsables de la obra } \\
\text { evaluada. El sistema registrará los estados del avance de } \\
\text { forma gráfica de cada docente. El evaluador dará a la obra } \\
\text { como aceptada, para actualización o negada según el caso. }\end{array}$ \\
\hline $\begin{array}{l}\text { Prioridad del requerimiento: } \\
\text { Alta }\end{array}$
\end{tabular}

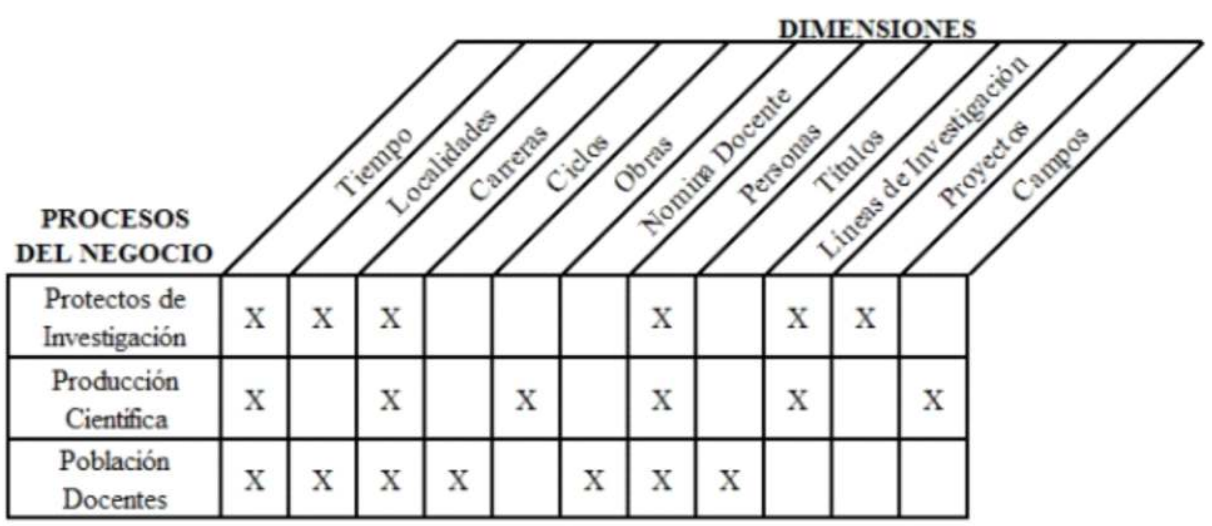

Figura 7. Matriz de Bus del Eje de Investigación de la UTN. 


\section{RESULTADOS}

Estos aspectos han sido evaluados mediante ENCUESTA a los stakeholder identificados de los cuales se ha obtenido los siguientes resultados:

Encuesta de valoración del Portafolio de la Dirección del CUICYT con sus análisis de Business Intelligence.

La encuesta fue aplicada a los actores directos quienes formaron parte de los stakeholder identificados, Entre los roles dentro de este grupo evaluado están la Directora del CUICYT, personal administrativo del CUICYT, Coordinadores de Investigación de cada facultad e Investigadores seleccionados. La muestra estuvo conformada por 20 personas en total.

A continuación, se presenta la encuesta con cada resultado.

1.- ¿Qué valor de aceptación tienen para usted el sistema de Business Intelligence para la gestión del eje de Investigación de la UTN?

\begin{tabular}{|c|l|}
\hline Valor & \multicolumn{1}{|c|}{ Opción } \\
\hline 1 & Muy en desacuerdo \\
\hline 2 & Discrepo \\
\hline 3 & Indeciso o incierto \\
\hline 4 & De acuerdo \\
\hline 5 & Totalmente de acuerdo \\
\hline
\end{tabular}

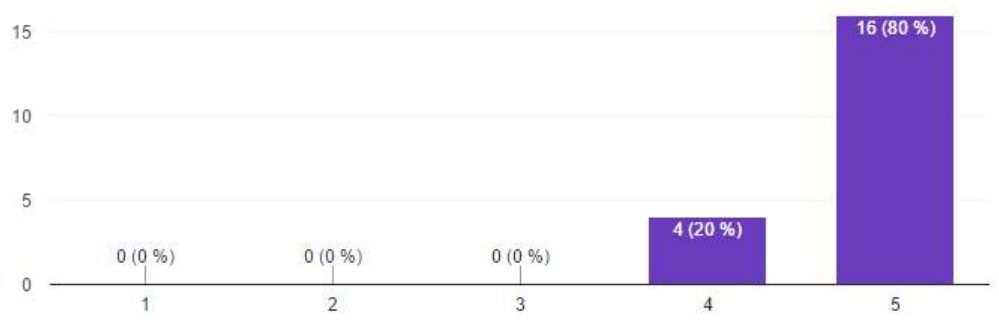

2.- ¿En relación a las siguientes tareas iqué nivel de ventaja se obtiene al utilizar el portafolio de la Dirección del CUICYT con sus análisis de Business Intelligence? 


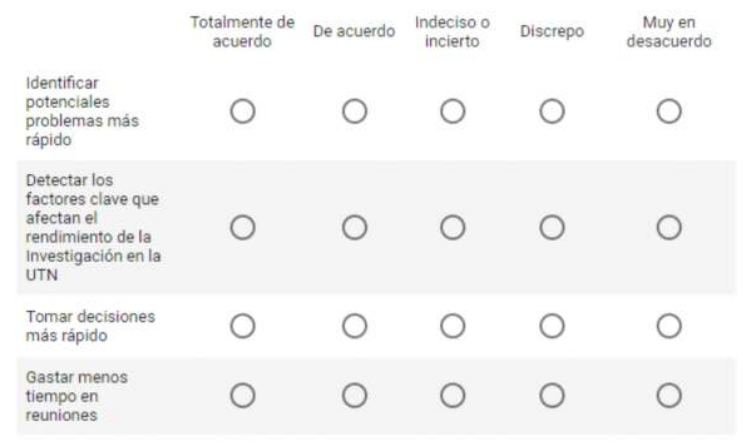

Resultado:

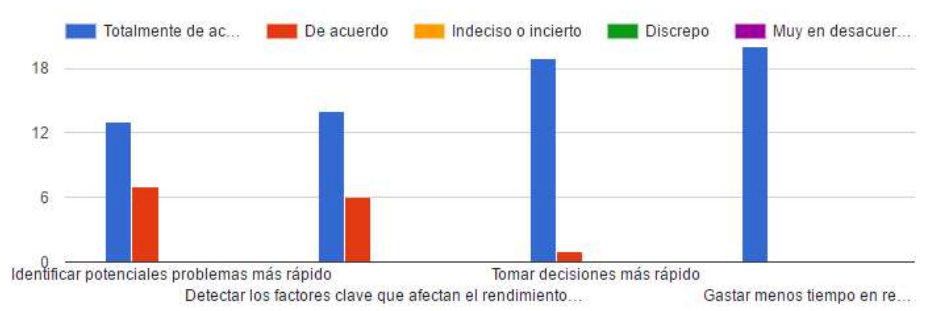

3.- ¿Dejar de usar el sistema de Business Intelligence sería una desventaja para mí?

\begin{tabular}{|c|l|}
\hline Valor & \multicolumn{1}{|c|}{ Opción } \\
\hline 1 & Muy en desacuerdo \\
\hline 2 & Discrepo \\
\hline 3 & Indeciso o incierto \\
\hline 4 & De acuerdo \\
\hline 5 & Totalmente de acuerdo \\
\hline
\end{tabular}

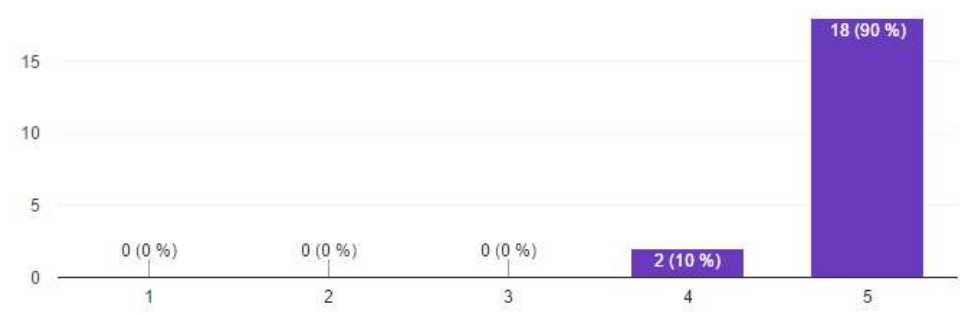




\section{ANÁLISIS DE RESULTADOS}

La encuesta aplicada refleja una alta aceptación de la solución implementada al problema identificado en esta investigación. Los usuarios del portafolio de la Dirección del CUICYT han encontrado en esta una herramienta imprescindible a la hora de evaluar el desarrollo de la Investigación de cada dependencia académica responsable. Los tiempos de generación de informes solicitados por las autoridades institucionales no tienen comparación por el simple hecho que no existía al nivel que ahora lo tienen, siendo por esto que no se ha hecho ninguna comparativa en ese enfoque. Los usuarios ven en la herramienta de BI una alternativa a la hora de identificar factores claves que puedan comprometer el puntaje de Investigación, esto en una próxima evaluación externa por parte de los organismos de evaluación y control gubernamentales, comprometiendo seriamente el avance y categorización de la Universidad considerando que el puntaje en Investigación es alrededor del 20\% del total de la evaluación.

\section{CONCLUSIONES}

La identificación de los indicadores claves para la gestión del CUICYT, ha permitido implementar un Dashboard que permita de primera mano mostrar el estado actual del eje de investigación de la UTN. Esto se ha logrado orientando los requisitos del diseño del almacén de datos de acuerdo a los objetivos estratégicos, visión y misión del CUICYT, permitiendo asíalinearse a la estrategia del negocio.

Para la Dirección del CUICYT, el resultado fue de gran impacto el tener un sistema informático que consolide toda la información estratégica del área en un solo cuadro de mando; y permita de una forma sencilla evaluar el estado actual del eje de Investigación de la UTN en cada uno de sus principales indicadores de gestión.

De igual forma el tener un portafolio de la Dirección del CUICYT que integre todas estas funcionalidades antes descritas ha sido de gran ayuda en la gestión, esto para la Directora actual del CUICYT y demás autoridades y coordinadores de investigación que tienen acceso a este portafolio.

También, ha permitido delegar funciones a los coordinadores de investigación en cada facultad, siendo estos coordinadores usuarios que se benefician al tener acceso a la información clave de Investigación en la UTN, lo que permitirá que aporten a la toma de decisiones y el trabajo se vuelva más colaborativo, todo con el fin de mejorar cada día los indicadores claves de Investigación que hoy en día para las IES se ha vuelto prioridad número uno en su gestión general. 


\section{REFERENCIAS BIBLIOGRÁFICAS}

[1] Bustinza O., Perez M., Ruiz A. (2013), Influencia de una cultura organizativa orientada hacia la calidad en los beneficios de la implantación de un sistema de gestión de recursos ERP, Intangible capital, volumen 9, 126-152.

[2] Chiesa, F. (2004). Metodología para selección de sistemas ERP. Reportes Técnicos en Ingeniería del Software, 6(1), 17-37.

[3] Díaz Barriga Arceo, F., Romero Martínez, E., \& Heredia Sánchez, A. (2012). Diseño tecnopedagógico de portafolios electrónicos de aprendizaje: una experiencia con estudiantes universitarios. Revista electrónica de investigación educativa, 14(2), 103-117.

[4] Barberà, E., \& Gregori, E. B. (2008). El estilo e-portafolio (Vol. 19). Editorial UOC.

[5] Oracle, C. (2009). ¿Qué es Inteligencia de Negocios?, 1-6. Retrieved from http://www.oracle.com/ocom/groups/public/@otn/documents/webcontent/317529_esa.pdf

[6] Torres, M., \& Ravelo, R. (2016). Comparing Tabular And Multidimensional Model In A Real BI Solution, 14(7), 3393-3399.

[7] A. Harrison, private communication, May 1995.

[8] Gartner. (15 de Enero de 2013). BI Competency Center Is Core to BI Success. Obtenido de http://www.gartner.com/resources/116400/116413/bi_competency_center_is _ core_116413.pdf

[9] Tableau Software. (2017). Mission. [online] Available at: https://www.tableau.com/about/mission\#7eHfyGXDqDSLlvZh.99 [Accessed 28 Apr. 2017].

[10] Oracle. (2013). Oracle Business Intelligence Suite Enterprise. Oracle White Paper, (April), 1-63. Retrieved from http://www.oracle.com/technetwork/middleware/bi/bi-foundationsuite-wp-215243.pdf?ssSourceSiteId=ocomen

[11] Gonzáles, Y., \& Rosales, M. (2014). Mercados de datos para el análisis estadístico de la información. 3C Tecnología, 3, 8-20.

[12] Caverol, J. M., Marcosl, E., Rey, U., \& Carzos, J. (2001). Metodologia para el DiseRo de Alnlacenes de Datos Etapa de Modelado Conceptual.

[13] P. Vassiliadis, A. Simitsis, P. Georgantas, M. Terrovitis, y S. Skiadopoulos, «A generic and customizable framework for the design of ETL scenarios», Information Systems, vol. 30, n⿳․․ 7, págs. 492-525, Nov. 2005.

[14] Di Tria, F., Lefons, E., \& Tangorra, F. (2016). Cost-benefit analysis of data warehouse design methodologies. Information Systems, 63, 47-62. https://doi.org/10.1016/j.is.2016.06.006

[15] Eric Ismael Leonard Brizuela, I., \& Castro Blanco, Y. (2013). Metodologías para desarrollar Almacén de Datos. Methodologies to develop Data Warehouse. Revista de Arquitectura E Ingeniería. 2013, vol.7 no.3, 77. 
Marco Carlozama, Lourdes Yépez, Jorge Caraguay

[16] Castelán, L., \& Ocharán, J. (2012). Diseño de un Almacén de datos basado en Data Warehouse Engineering Process (DWEP) y HEFESTO. Universidad Veracruzana, 10. Retrieved from http://www.uv.mx/mis/files/2012/11/Diseno-de-un-Almacen-de-datos.pdf

[17] Leal, A. C., Trujillo, J., \& Mazón, J. N. (2013). A business-oriented approach to data warehouse development. Journal of Information Systems, 33 (1), 59-65.

[18] Cravero, A., \& Sepulveda, S. (2016). Methodologies, techniques and tools for OLAP design: A Systematic Mapping Study. IEEE Latin America Transactions, 14(2), 943-950. https://doi.org/10.1109/TLA.2016.7437244. 